\title{
IMPACT OF COVID-19 ON BREAST CANCER TUMOR SIZE AT DIAGNOSIS
}

\author{
Andrei Alves de Queiroz, Gustavo Machado Badan¹, Marilucia Batina Fernandes Moreira, \\ Amanda Neves Machado ${ }^{1}$ \\ ${ }^{1}$ Hospital Samaritano de São Paulo - São Paulo (SP), Brazil.
}

Introduction: In 2020, COVID-19 affected the whole world, determining a pandemic situation, with recommendations for social isolation and lockdown. In the state of São Paulo, the shutdown of non-essential services was determined on March 22, 2020. Medical appointments and routine imaging exams were postponedand little is known about the impact on screening delay and the diagnosis of neoplasms. Considering that survival and cure of breast cancer are directly related to an early diagnosis, the size of these malignant tumors can be used in researching the delay in breast cancer diagnoses. Objectives: To evaluate the impact of the pandemic on the size of breast cancer in histological diagnosis, as well as on the number of diagnostic procedures performed at our hospital. Methods: Through a retrospective, analytical and crosssectional study, we analyzed data regarding tumors with histological results of malignancy of core needle breast biopsies guided by ultrasound performed at a private hospital in São Paulo between January 1, 2019 and December 31, 2020. The mean tumor sizes were compared to identify differences between prepandemic and pandemic periods. The prepandemic period (PRE) was established between January 1, 2019 and March 31, 2020, and the pandemic period (PAN) was considered from April 1 to December 31,2020. Based on the sample size, this study has the power of $80 \%$ to detect a variation of $1 \mathrm{~cm}$ in the mean tumor size. Results: A total of 493 core needle biopsies were identified in 443 patients. A total of 103 (20.1\%) biopsies in 94 patients were malignant. In the PAN group, 36 cases of cancer were diagnosed ( 4 cases/month), while the PRE group diagnosed 67 cases ( 4.5 cases/month). The mean size of PRE tumors was $1.66 \mathrm{~cm}$, while in PAN tumors it was 2.21, showing a difference of $0.55 \mathrm{~cm}$ without statistical significance (95\%CI $0.12-1.21 ; \mathrm{p}=0.12)$. When considering staging (TNM Tumor, Node, Metastases), the stages T1, T2 and T3 had no significant difference among the groups ( $\mathrm{p}=0.12)$. The age at diagnosis of malignant lesions ranged from 30 to 82 years in the PRE period, and from 34 to 85 years in the PAN period. The mean age of patients with malignant lesions diagnosed in PAN was higher than PRE, without statistical significance (59.2 vs 56.0; $\mathrm{p}=0.30)$. Despite the fewer biopsies performed in both periods, $(\mathrm{p}<0.001)$, there was no statistical difference in the number of biopsies with malignant results $(\mathrm{p}=0.18)$, since there were proportionally more diagnoses of malignancy in the PAN period (28.6\% vs. 18.3\%; RR 1.14; 95\%CI 1.01-1.29; $\mathrm{p}=0.02$ ). Conclusions: Although the pandemic affected breast cancer screening, no statistically significant increase in the mean size of tumors has been diagnosed in this service so far. 